\title{
An Interactive Exploratory System with Real-Time Preference Elicitation
}

\author{
Panagiotis Papadakos, Yannis Tzitzikas and Dafni Zafeiri \\ Institute of Computer Science, FORTH-ICS, GREECE, and \\ Computer Science Department, University of Crete, GREECE \\ Email: \{papadako|tzitzik|dzafiri\}@ics.forth.gr
}

Keywords: Exploratory Search, Multi-Dimensional Information Spaces, Preferences, Decision Making

Introduction Current proposals for preference-based information access [4] seem to ignore that users should be acquainted with the information space and the available choices for describing effectively their preferences. Furthermore, users rarely formulate complex (preference or plain) queries, because it is a laborious and difficult task for them. We will demonstrate a system for interactive exploration of multi-dimensional and hierarchical information spaces, enriched with actions that allow users to dynamically express their preferences, based on the preference framework described in [5]. Specifically, the system supports progressive preference elicitation, inherited preferences with scope-based resolution of conflicts, and preference composition over multi-dimensional and hierarchical information spaces. We argue that such functionality can ease the interaction and speed up the restriction of the focus to those parts of the information space that the user is interested in.

Preference enabled exploration The proposed interaction model can be implemented over a variety of exploratory methods like the interaction paradigm of Faceted and Dynamic Taxonomies (FDT) [2,3]. We will demonstrate an implementation over a system for browsing and exploring RDF sources, described in detail in [1] (the fuzzy aspect is ignored). This system uses Jena ${ }^{1}$, which is a Java framework for building Semantic Web applications. Our information base holds about 4036 cars and trucks, which are described by classes like Manufacturer and Drive_System (hierarchically organized), Vehicle_Type, and Transmission, as shown in Fig. 1. In this figure, continuous arrows denote subClass Of relationships while dashed arrows denote typeOf relationships.

The architecture of the system and its components is given in Figure 2. The preference actions are offered through HTML 5 context menus ${ }^{2}$ and AJAX, which are enacted by right clicking in the browser window. The user is able to order classes, subclasses and objects using best, worst and prefer to actions (i.e. relative preferences), or actions that order them lexicographically or based on their count values. Regarding objects, since their number can be very large,

\footnotetext{
${ }^{1}$ http://jena.apache.org/

${ }^{2}$ Available only to firefox 8 and up.
} 


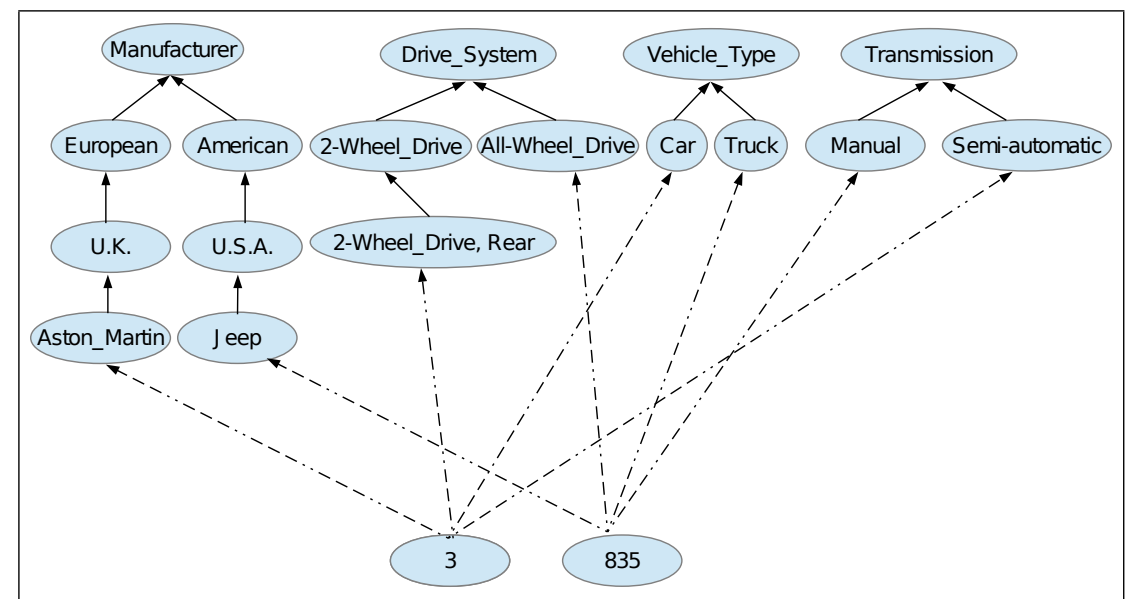

Fig. 1. The RDF Knowledge Base

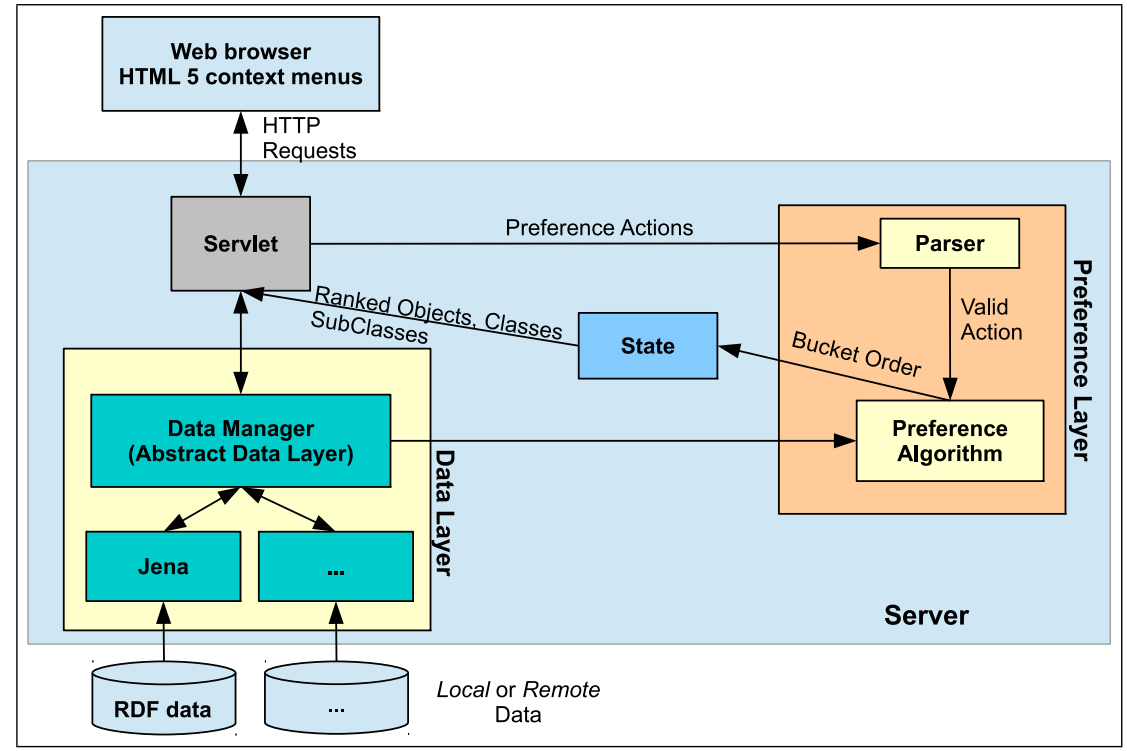

Fig. 2. The architecture of the system

the user is able to define a threshold, so that preferences are applied only when the number of objects is reduced under this specific threshold ${ }^{3}$. Furthermore, he can compose object scoped preferences anchored to classes, using Priority, Pareto and Pareto Optimal compositions. Composition is offered by defining the appropriate composition mode and selecting classes through the appropriate

${ }^{3}$ The user can reduce the number of objects by navigating over the classes, subclasses, and objects and restricting his focus. 
classes' context menus. Finally, the user is able to store and load his preferences, since exploration is a time depth process.

A number of screenshots of the system with the available options and indicative preference actions is shown below. Figure 3 depicts the available system options (i.e. composition, threshold, session store and load) and the expression of a simple preference action. Figure 4 shows how we can define a relative preference action anchored to terms that affects the order of objects. Figure 5 shows how to order all objects lexicographically, and finally Figure 6 shows how the user can easily express more complicated actions, like Pareto composition.

This demonstration focuses on the flexibility of the provided preference actions and how with a few actions the user can select the desired car from an information base of 4036 cars.

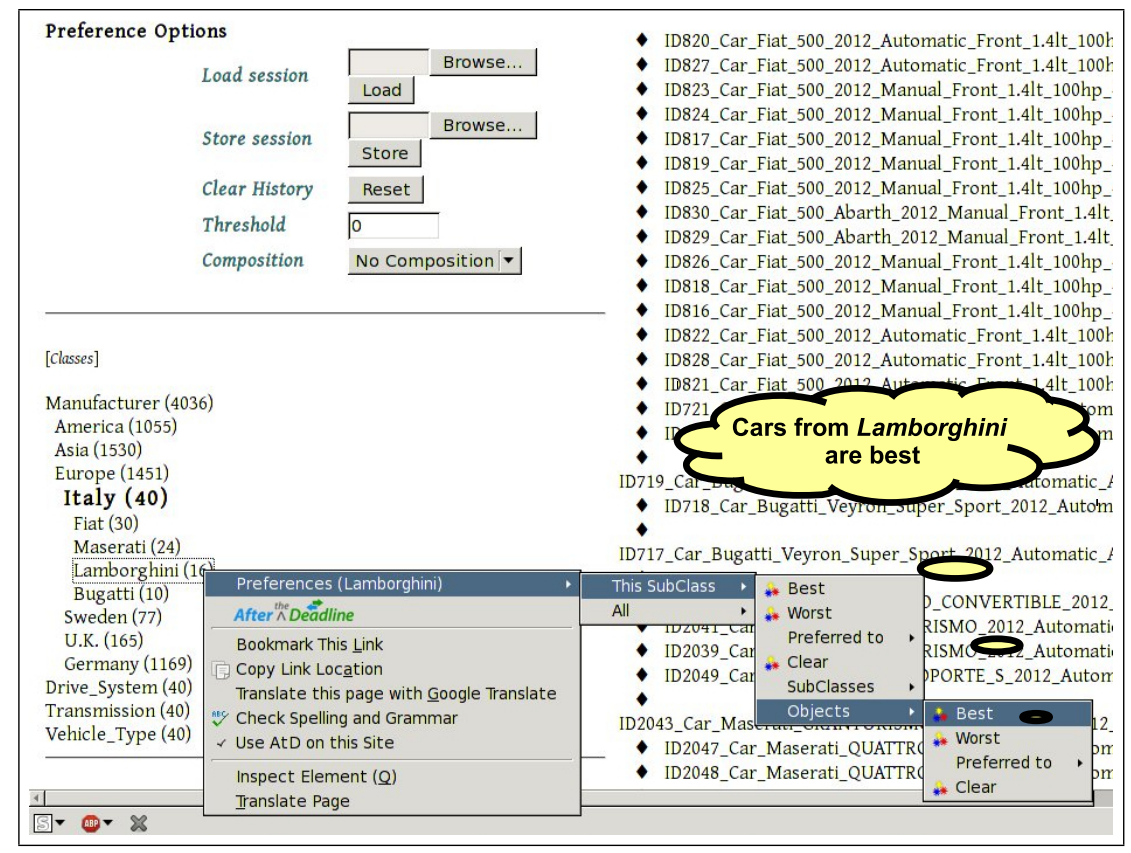

Fig. 3. Overview of the system options and a simple preference action (i.e. Cars from Lamborghini are best)

\section{Acknowledgements}

This research has been co-financed by the European Union (European Social Fund - ESF) and Greek national funds through the Operation Program "Education and LifeLong Learning" of the National Strategic Reference Framework (NSRF) - Research Funding Program: Herakleitus II. Investing in knowledge society through the European Social Fund. 


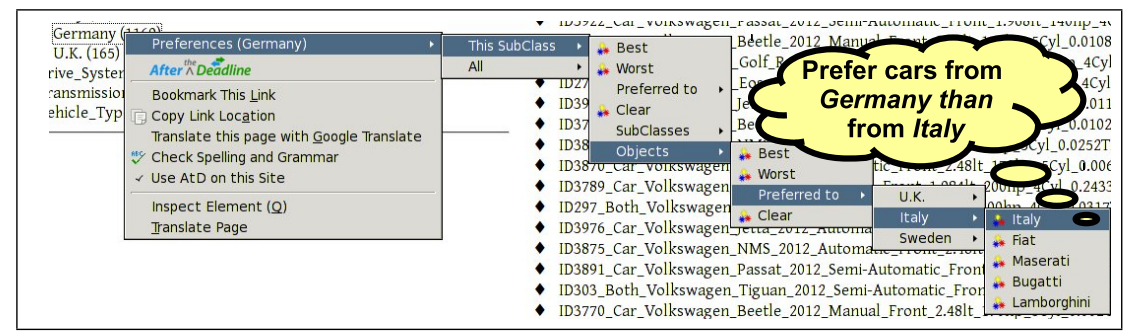

Fig. 4. Action to Prefer German to Italian cars

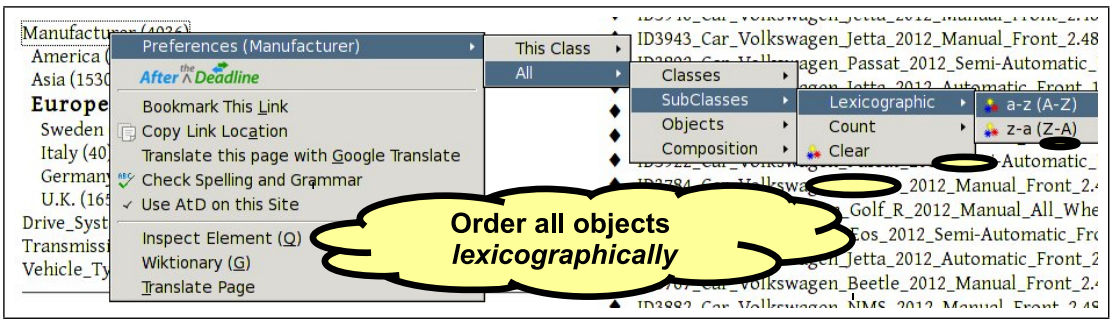

Fig. 5. Order all objects lexicographically in ascending order

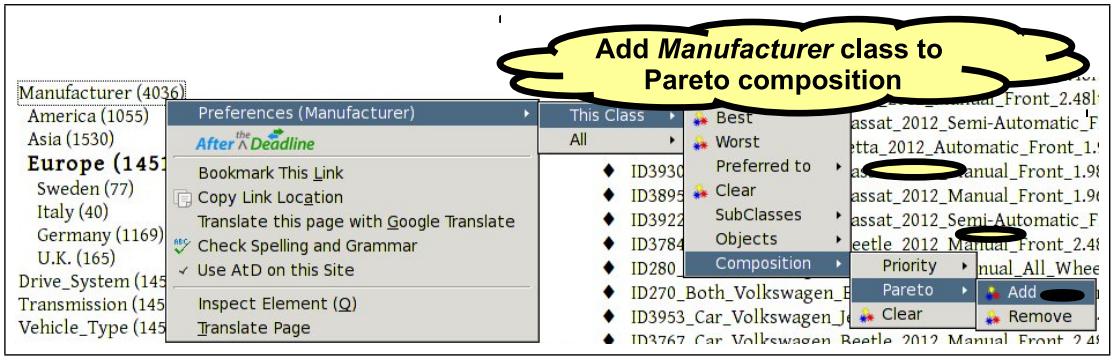

Fig. 6. Add Manufacturer class to Pareto composition

\section{References}

1. N. Manolis and Y. Tzitzikas. "Interactive Exploration of Fuzzy RDF Knowledge Bases". In Procs of the 8th Extended Semantic Web Conf. (ESWC'11), Heraklion, Greece, 2011.

2. P. Papadakos, N. Armenatzoglou, S. Kopidaki, and Y. Tzitzikas. "On Exploiting Static and Dynamically Mined Metadata for Exploratory Web Searching". Knowledge and Information Systems, 30(3):493-525, 2012.

3. G. M. Sacco and Y. Tzitzikas, editors. "Dynamic Taxonomies and Faceted Search: Theory, Practise and Experience". Springer, 2009.

4. K. Stefanidis, G. Koutrika, and E. Pitoura. "A Survey on Representation, Composition and Application of Preferences in Database Systems". ACM Trans. Database Syst., 36:19:1-19:45, August 2011.

5. Y. Tzitzikas and P. Papadakos. "Interactive Exploration of Multi-Dimensional and Hierarchical Information Spaces with Real-time Preference Elicitation". Fundamenta Informaticae. (Accepted for publication in 2012). 